\title{
Lymphoepithelial Carcinoma with Special Reference to Nasopharynx-A Study of 35 Cases
}

\author{
Dr. T. Subachitra ${ }^{1}$, Dr. A. Sundaram ${ }^{2}$, Dr. Chithra Srinivasan ${ }^{3}$, \\ Dr. R. Sathyalakshmi ${ }^{4}$ \\ ${ }^{I}$ (Department of Pathology, Stanley Medical College,The Tamilnadu Dr.MGR Medical University,India) \\ ${ }_{2}^{2}$ (Department of Pathology, SRM Medical College,SRM University,India) \\ ${ }_{3}^{3}$ (Department of Pathology, Saveetha Medical College,Saveetha University,India) \\ ${ }^{4}$ (Department of Pathology, Stanley Medical College,The Tamilnadu Dr.MGR Medical University,India
}

\begin{abstract}
Lymphoepithelial carcinoma is an undifferentiated neoplasm arising in the nasopharyngeal mucosa.The incidence of Lymphoepithelial carcinoma in the Indian subcontinent is not well documented.The aim of the present study compares the incidence, age,sex distribution, clinical presentation, and relationship of $E B V$ virus and LEC in sporadic cases of LEC from India world statistics. And also the role of LatentMembraneProtein-1in diagnosing the EBV association.Malignant lesions with prominent lymphoepithelial component were taken for the study.Pancytokeratin,lymphoma markers were used to confirm the histopathological diagnosis.LMP-1 was used to demonstrate EBV in tissue sections.It was observed that nasopharynx being the commonest site for LEC.It showed male preponderance with bimodal age distribution.Intense staining of LMP-1was demonstated in LEC of nasopharynx than other sites. Hence confirming the strong EBV association in LEC of nasopharynx. It was also observed that LEC with early lymphnode metastasis showed intense LMP-1 staining.Thus confirming the progressiveness and metastatic potential of LMP-1 expression in EBV associated LEC when compare to EBV negative LEC.

Hence LMP-1 proves to be a useful IHC marker in establishing the metastatic potential of LEC and the need for early diagnosis and treatment of $L E C$.
\end{abstract}

Key Words: Lymphoepithelial carcinoma,Nasopharynx,Latent membrane protein-1,EBV virus

\section{Introduction}

Lymphoepithelial carcinoma is an undifferentiated malignant neoplasm arising from the nasopharyngeal mucosa that show evidence of squamous differentiation.LEC of nasopharynx is remarkable for its striking geographic difference in its incidence as well as the strong association of Epstein-Barr virus.AlthoughLEC is rare world wide, it is one of the most common cancers in SouthEast Asian countries and in China.the incidence there is $20-50$ per 100000 individuals ${ }^{1}$ It can arise in variety of sites,head and neck mucosal sites,salivary gland,lung,esophagus and thymus. Very few studies of LECare documented in India.LEC is generally rare in India as compared to SouthEast Asian countries, with the exception of some northeast states ,mainly Nagaland. ${ }^{2}$

The present study from India aims at comparing the incidence, age and sex distribution,clinical features,and its EBV association with world statistics. Strong EBV association with LEC of nasopharynx has been studied using Latent Membrane Protein-1as an IHC marker, It also tries to establish the high levels of LMP-1expression with the early metastatic potential of EBV associated LEC.

\section{Materials And Methods}

The study was conducted from the period of 2007-2010,both prospectively and retrospectively. All the biopsy samples received in the department of pathology,Stanley Medical College,were analysed.Total $\mathrm{f} 58$ cases with malignant lesions showing prominent lymphoepithelial component were taken for the study.Samples taken from the nasopharynx were given special reference.

Samples are routinely processed and stained with Haematoxylin and Eosin.Epithelial tumors showing prominent lymphoplamocytic infiltration were confirmed by Pancytokeratin and lymphoma markers. Suitable sections were selected for IHC.Slides were coated with chrome alum, and subjected to antigen retrieval using microwave technique with TRIS-EDTA buffer solution.Slides were then treated by HRP(Horse radish peroxidise)polymer technique.

Pancytokeratin positive tumors were stained with LMP-1(CSI-4 antibody)to analyse the EBV association. LMP1 was used in all cytokeratin positive samples using Hodgkins lymphoma as a control.Immunostaining was scored on the basis of positive tumor cells and the relative immune staining intensity. 
The following grading system was adapted to score the number of positive cells

Scoring method ${ }^{3}$

0 -none seen in the section

1 -presence of rare positive cells

$2-26$ to $50 \%$ positive cells

$3-51$ to $75 \%$ positive cells

4-76 to $100 \%$ positive cells

Immunostaining intensity

0 -none

1-weak

2-moderate

3-intense

\section{Observation And Results}

Out of 58 cases, 35 cases were diagnosed as lymphoepithelial carcinoma(.fig1-4),21 cases of poorly differentiated carcinoma.35 cases were positive for Pancytokeratin. (fig 5). and CD 45,CD20,CD3 were positive in infiltrating lymphocytes.(fig5)

13 cases showed LMP-1 positive staining with 5 cases showing intense staining.fig (6).

Majority of cases belong to younger age group(10-20) and 61-80 years of age showing bimodal distribution.Nasopharynx was the commonest site .Most of the cases presented with nasal obstruction and nasal discharge, 4 cases presented with cervical node metastasis as initial clinical manifestation.

Younger age group showed high levels of LMP-1.Also the LEC of nasopharngeal origin showed intense staining for LMP-1.

\section{Discussion}

Nasopharyngeal carcinoma is often difficult to diagnose because of nonspecific nature of its clinical symptoms and the difficulty in visualizing the nasopharyn $\mathrm{x}^{4}$.Submucosal primary lesions often escape endoscopic examination.Most of the tumors remain undiagnosed until they present as metastasis to cervical lymphnodes, often without overt pathology at the primary site.FNAC will be helpul in such cases. One such case was diagnosed with FNAC ,in cervical node and it is correlated with HPE diagnosis.

Koppiker et al,have reported that $5 \mathrm{yr}$ actual disease free survival of Indian patients is $13 \%$ which indicates the need for an early diagnosis and treatment strategies to improve survival ${ }^{5}$.Early detection of LEC should improve cure rate and reduce morbidity and metastasis. This requires an effective screening system.

Out of 35 cases of lymphoepithelial carcinoma10 cases were found in the younger age group (10-20 )yrs and 8 cases in the older age group(61-80) yrs,Balakrishnan et al $^{6}$ observed bimodal age distribution with peaks in age group(15-24) and (45-54) yrs from India.

This present study confirms the bimodal distribution except for the peak in 60-80 yrs of age group in contrast to 45-54 age groups in the study of Balakrishnan et.al.This study also confirms the male preponderance of lymphoepithelial carcinoma as in previous studies

Most of the patients in this study, presented with nasal obstruction and nasal bleeding (83\%), three of them presented with cervical node metastasis as the intial manifestation.

Pan cytokeratin positive cases were selected for immunostaining with latent membrane protein-1 and it was found that LMP-1 was associated with $79 \%$ of the cases with nasopharyngeal origin.these results are consistent with Raab-Taub et al and Chang et al ${ }^{7,8}$.

Of LEC among other sites,salivary gland LEC strong association with LMP-1weaker and moderate staining in pyriform fossa and posterior 1/3of tongue respectively.LEC in other than these sites showed negative results which are consistent with Bijan Khademi et al $^{9}$ indicating LMP-1 association is more specific for LEC of nasopharyngx/

This present study also shows that higher levels of LMP-1 expression was associated with younger age grop $(75 \%)$,confirming the earlier study by Abdel Majiid Khabir et al.In contrast to his study higher levels of LMP-1 expression associated with lymphnode metastasis.

As per literature LMP-1 potentiates a variety of signalling pathways including the nuclear factor kb,mitogen activated protein kinase and phosphotidylinositol-3 kinase Alt pathways and involved in angiogenesis which is a key step in tumor growth ,invasion and metastasis ${ }^{10}$.

LMP-1 protein is essential for EBV mediated immortalization of B lymphocytes and its expression has been shown to inhibit the terminal differentiation of keratinocytes, thus providing a possible explanation for the lack of differentiation of most EBV associated epithelial tumor cells ${ }^{11}$.

In addition to oncogenesis, LMP-1 is suggested to be revelent to the metastatic property of LEC $^{12}$. Studies also report that LMP-1 positive NPCS show a more progressive attitude and an increased 
Lymphoepithelial Carcinoma with Special Reference to Nasopharynx-A Study of 35 Cases

tendency towards lympnode metastasis than LMP-1 negative NPCs,Studies suggest that induction of c-met proto-oncogene by LMP-1 is mediated by activation of Ets-1 transcription factor which leads to upregulation of cell motility considered to be an essential factor in the steps of metastasis ${ }^{13},{ }^{14}$.

In this present study 4 cases presented with cervical node metastasis, were associated with high levels of LMP-1 expression.Also a 13 yr old boy with LEC who presented with vertebral region metastasis within one year of the diagnosis of the primary tumor and was found to be associated with high levels of LMP-1 expression.

Interestingly, according to Padmanathan and collegues LMP-1 was detected in all preinvasive NPCs that quickly developed into invasive NPCs ${ }^{15}$.Thus LMP-1 mediated enhancement of metastatic potential could be an early event in NPC.

To confirm, the association of EBV in agiven tumor,the virus must be detected within the tumor cells.EBV LMP-1, in usually positive in $30-40 \%$ of cases.It is usually less sensitive and specific than other molecular analysis.

However Abdel Majiid Khabir et al. observed in his study that no biopsy is completely devoid of LMP1 positive cells and he also suggested the use of S12 antibody which is more sensitive in staining tissue section than CSI-4 antibody.In this present study we used CSI-4 antibody for detecting the presence of EBV in tissue sections .

Dietz et al concluded that tyramin augmented IHC for LMP-1 staining was found to be clearly positive in samples previously showed negative staining with conventional IHC.Both the studies showed 100\%LMP-1 expression.

Conclusion: Despite the limitations of LMP-1,its simplicity,applicability to paraffin sections and its use as an indicator of progressiveness of the tumour has made it an attractive ancillary method for early diagnosis of LEC.

\section{References}

[1]. Muhitranga A,Tanunyitthawongese C,Pornthanakarem W,KerekhanjanarongV,Yenrundi S,et al.genomic alteration in nasopharyngeal carcinoma;Epstein Barr virus infection.BritJ cancer1997;76:770-6

[2]. James E,Marks M.D,Jessi Linn Philips,Herman R Menck et al nasopharyngeal carcinoma in India.Cancer 1998;83:582-588

[3]. Abdel Majid Khabir,Hela Karry,Sandrine Rodriguez,Mathieu Rose et al EBV Latent Membrane Protein -1 Abundance correlates with patent age but not with the metastatic behaviour in North African nasopharyngeal carcinoma.Virology J 2005;2:39

[4]. Zong YS,Sham JS,Ng MH,Ou XT,Guo YQ,Zheng SA et al,Immunoglobulin A against capsid antigen of Epatein Barr virus and Indirect mirror examination of the nasopharynx in the detection of Nasopharyngeal cancer in a high risk population.J Natl Cancer Inst 1999;91:796-800

[5]. Koppikar SB,Advain SH,GopalR,Dinshaw KA,Pandle SC,Nair CN,et al, Nasopharyngeal carcinoma in India :End result analysis(1980-1984).U Surg Oncol 1988:39:179-82.

[6]. Balakrishnan V,Gangadharan P,and Nagaraj RaoD. Epidemiological tiologicalfactors associated with nasopharyngeal cancer ICMR bulletin 2003 vol.33 no.9.

[7]. Raab-Traub N, Flynn K,Pearson G Huang A, Levine P, Lanier A etal, The differential form of Nasopharyngeal carcinoma contains Epstein Barr virus DNA. Int J Cancer 1987; $39: 25-9$

[8]. Chang YS,Tyan YS,Liu ST,Tsai MS,Pao CC.Detection of Epstein-Barr virus DNA sequences in nasopharyngeal carcinoma cells by DNA amplication.J.Clin Microbiol 1990;28:2398-402.

[9]. Bijan Khademi,Jalal Mahmoudi,Ahmad Monobati.BehZed Maghsoudi, et al .Molecular diagnosis of nasopharyngeal carcinoma using detection of Epstein Barr virus Latent membrane protein-1 gene in cervical node metastatic lymphnodes.Am J Otolaryn 2009;30:95-100.

[10]. Murono,S.Yoshizaki,T,Sato,H.,Takeshita,H.Furukawa,M and Pangano,J.S.Aspirin inhibits tumor cell invasiveness induced by Epstein Barrvirus latent membrane protein-1 through expression of matrix metalloproteinase-9 expression.Cancer Res,60;2555561.2000.

[11]. Dawson C.W, Rickinso A.B snd Young L.S.Epstein Barr virus latent membrane protein inhibits human epithelial cell differentiation.Nature(lond)344;777-780,2000.

[12]. Horikawa T,Shen TS,Takeshika H,Sato H,Purukawa M,Yoshizaki T Induction of C-met Proto oncogene by EBV LMP-1and the correlation with cervical lymphnode metastasis of nasopharyngeal carcinoma Am J Pathol 2001,59:27-33.

[13]. Henderson S.,Row.M.Gregory C., Croom-Carter,D.Wanf.F,Longnecker.R.Kieff,E.,and Rickinson AB,Induction bcl2 expression by the .Epstein Barr virus latent membrane protein protects infected B cells from programmed cell death,Cell,65;1107-1115.

[14]. C.N Ian X,Guo,B.Cao EJ,Kort CC,Lee J Chen,L M,Wang WY Mai HQ,Min MH Hong,et al, latent membrane protein leve correlates with survival in patients with late stage nasopharyngeal carcinoma.Cancer Res.,2002;62(2):589-596.

[15]. Pathmanathan RU,Prasad U,Sadler R,Flynn,K,Raab Traub N;Clonal proliferations of cells infected with Epstein Barr virus in Preonvasive lesion related to Nasopharyngeal carcinoma. N Engl J Med 1955, (3)33:693-698.

[16]. PlazaG,Manzanal AI,Fogue L,Santon A,Martinez-Montero JC,Bellas C ,Association of Epstein Barr virus and nasopharyngeal carcinoma in Caucasian patients.2002;111:210-216

[17]. Dietz A,Logothetis C.A,Helbig M et al Prognostic impact of EBV related LMP-1, Histologic type and environmental factors in Nasopharyngeal carcinoma in german population. Onkologie 2004;27:345-350.

[18]. G Bar-Sela, A Kuten I,Minkov et al, Prevalance and relevance of EBV larency in nasopharyngeal carcinoma in Isarel.J Clin Pathol 2004;57(3):290-293. 
Age distribution of lymphoepithelial carcinoma

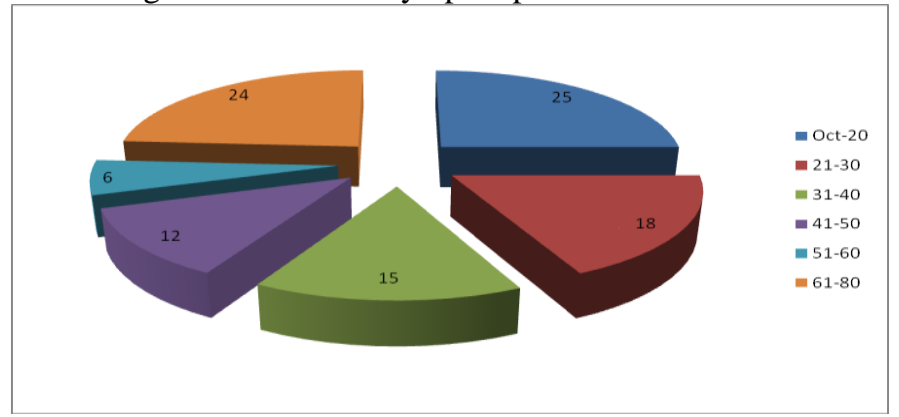

Clinical presentation

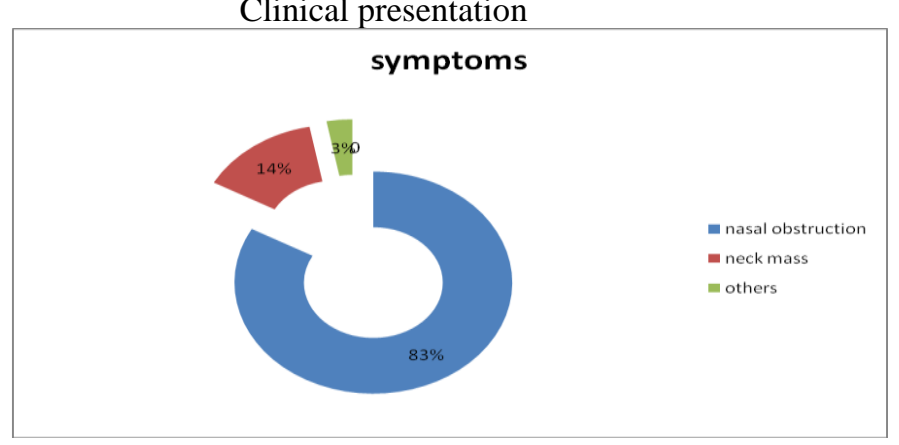

Clinical correlation of LMP-1 with age of the patients

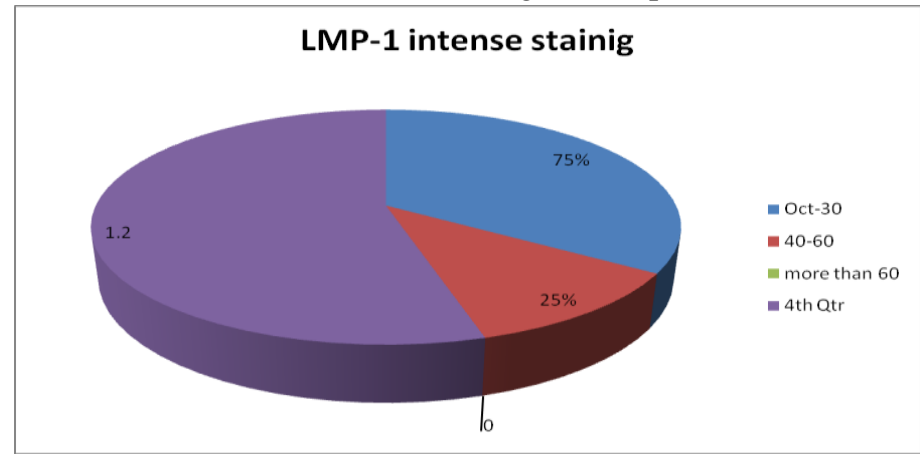

Clinical correlation of LMP-1 with site of LEC

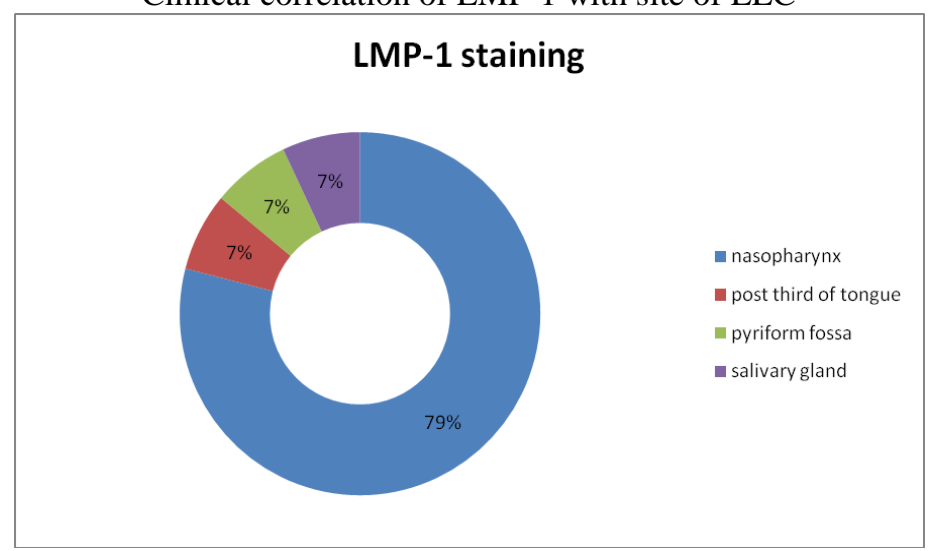


Lymphoepithelial Carcinoma with Special Reference to Nasopharynx-A Study of 35 Cases

Table no.1 Site distribution of LEC

\begin{tabular}{|l|l|}
\hline Site & No.of cases \\
\hline Nasopharynx & 26 \\
\hline Pyriform fossa & 2 \\
\hline Salivary gland & 1 \\
\hline Nasal cavity & 1 \\
\hline Larynx & 1 \\
\hline Posterior 1/3 of tongue & 1 \\
\hline Tonsil & 1 \\
\hline Hypopharynx & 1 \\
\hline Epiglottis & 1 \\
\hline Total & 35 \\
\hline
\end{tabular}

Table-2 Clinical correlation of LMP-1 with age of the patients

\begin{tabular}{|l|l|l|l|}
\hline \multirow{2}{*}{ Age } & LMP-1 & Meak \\
\cline { 2 - 4 } & Intense & Moderate & we \\
\hline $10-30$ & 4 & 2 & 3 \\
\hline $40-60$ & 1 & 2 & - \\
\hline More than 60years & - & 1 & 1 \\
\hline
\end{tabular}

Table -3

\begin{tabular}{|l|l|l|l|}
\hline Reference & Year of study & $\begin{array}{l}\text { No.of LMP-1 positive } \\
\text { samples }\end{array}$ & Tumor tissue source \\
\hline Plaza et al $^{16}$ & 2002 & $13 / 30$ & Paraffin block \\
\hline Dietz et al $^{17}$ & 2004 & $33 / 33$ & Paraffin block \\
\hline G Bar Sela et al $^{18}$ & 2004 & $6 / 45$ & Paraffin block \\
\hline Abdel Majiid et al & 2005 & $29 / 29$ & Paraffin block \\
\hline Present study & 2010 & $16 / 25$ & Paraffin block \\
\hline
\end{tabular}

Pictures

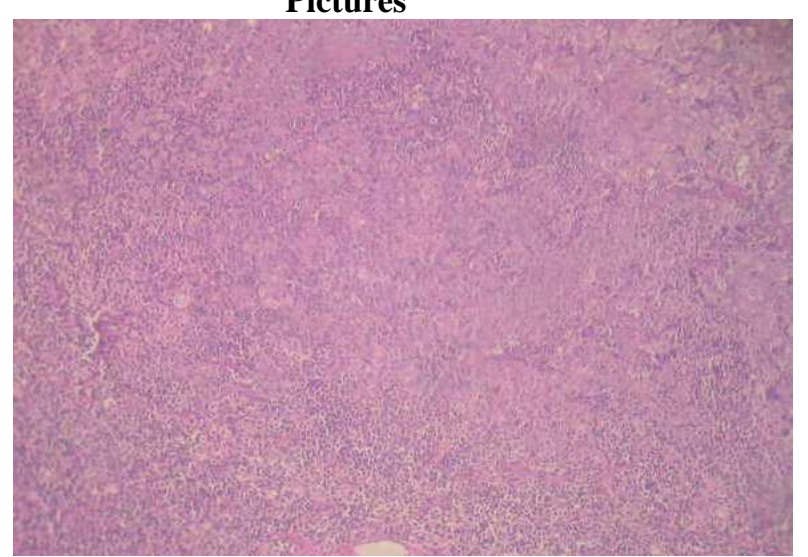

Figure -1 4x view of LEC showing sheets of tumor cells with interspersed lymphocytes

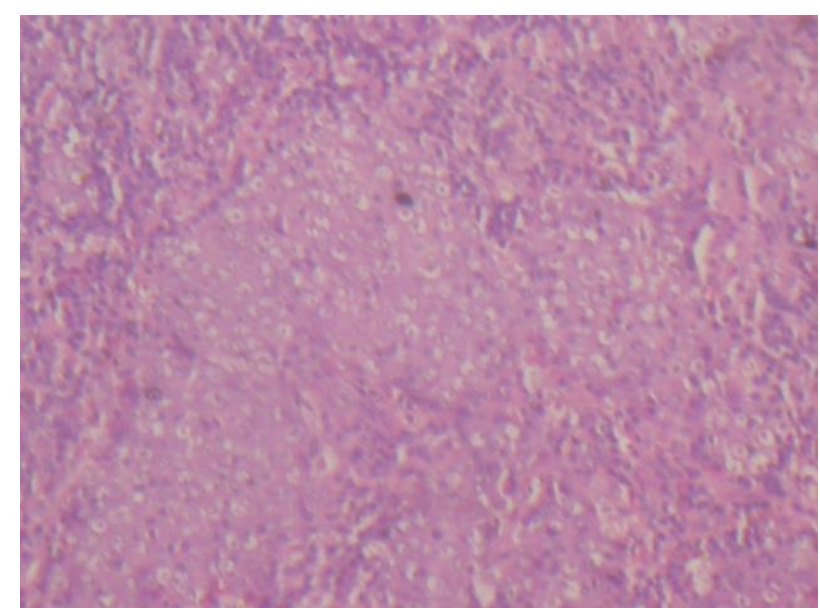

Figure: 2 10x view of tumor cells arranged in sheets. 


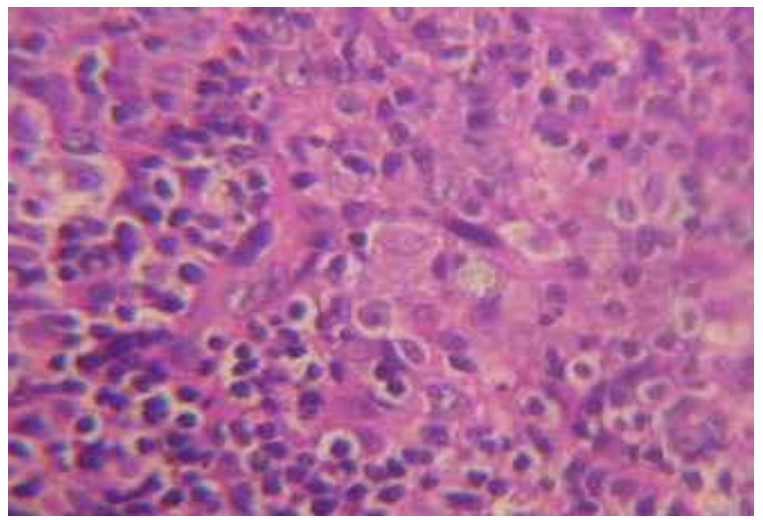

Figure-3 Polyhedral cells with vesicular nuclei and prominent nucleoli

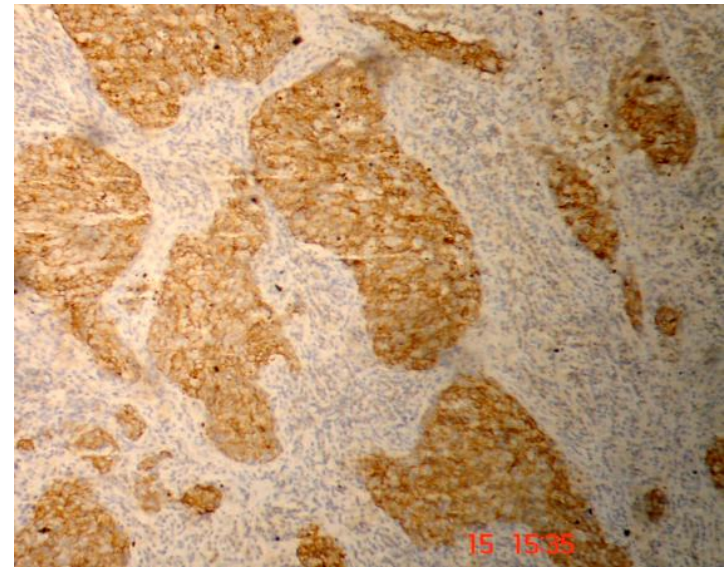

Figure-4 Cytokeratin positive epithelial cells

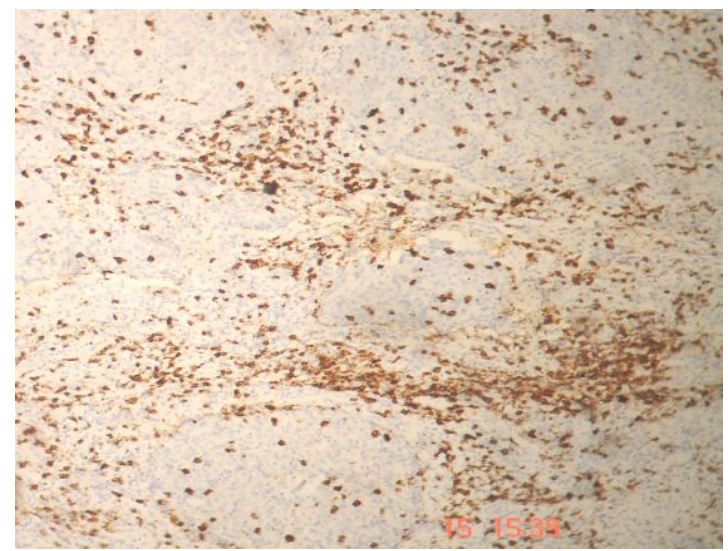

Figure-5 Non neoplastic lymphocytes positive for CD 45 immunostaining

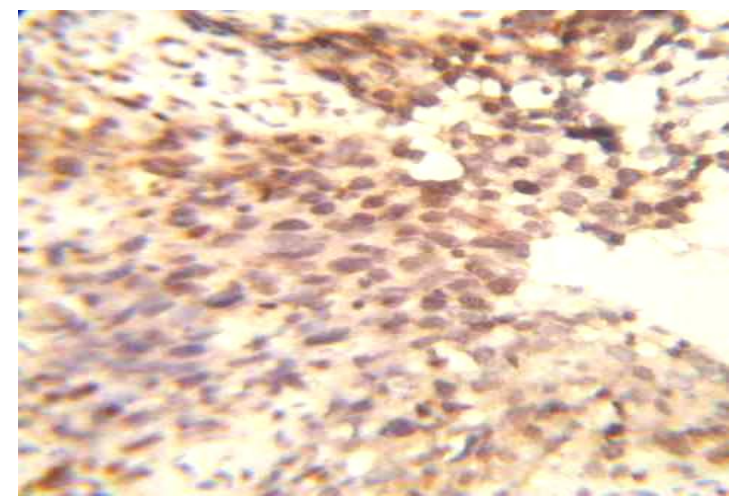

Figure 5 LMP-1 positive staining in tumour cells 